\title{
Levinson's theorem and scattering phase-shift contributions to the partition function of interacting gases in two dimensions
}

\author{
M. E. Portnoi* and I. Galbraith \\ Physics Department, Heriot-Watt University, Edinburgh EH14 4AS, United Kingdom
}

(Received 19 February 1998)

\begin{abstract}
We consider scattering state contributions to the partition function of a two-dimensional (2D) plasma in addition to the bound-state sum. A partition function continuity requirement is used to provide a statistical mechanical heuristic proof of Levinson's theorem in two dimensions. We show that a proper account of scattering eliminates singularities in thermodynamic properties of the nonideal $2 \mathrm{D}$ gas caused by the emergence of additional bound states as the strength of an attractive potential is increased. The bound-state contribution to the partition function of the $2 \mathrm{D}$ gas, with a weak short-range attraction between its particles, is found to vanish logarithmically as the binding energy decreases. A consistent treatment of bound and scattering states in a screened Coulomb potential allowed us to calculate the quantum-mechanical second virial coefficient of the dilute 2D electron-hole plasma and to establish the difference between the nearly ideal electron-hole gas in $\mathrm{GaAs}$ and the strongly correlated exciton/free-carrier plasma in wide-gap semiconductors such as $\mathrm{ZnSe}$ or GaN. [S0163-1829(98)09531-9]
\end{abstract}

\section{INTRODUCTION}

Two-dimensional (2D) systems play a central role in contemporary condensed matter physics. Novel phenomena such as the quantum Hall effect ${ }^{1}$ observed when a $2 \mathrm{D}$ electron gas at low temperature is subjected to a strong magnetic field, as well as practical developments based on quasi-2D systems, e.g., high-mobility field-effect transistors ${ }^{2}$ or semiconductor quantum-well lasers, ${ }^{3}$ have brought significant technological advances. Such devices are based on the quasiequilibrium response of the internal electron or electron-hole plasmas to an external stimulation. Hence it is essential to understand the fundamental quantum-statistical properties of such twodimensional interacting plasmas at finite temperatures.

One of the well-known differences between 2D and 3D nonrelativistic quantum mechanics is the presence of at least one bound state for any symmetric attractive potential in two dimensions. This bound state, with binding energy $E_{b}$, brings a nonvanishing contribution of $\exp \left(E_{b} / k_{B} T\right)$ to the two-body part of the partition function even if the interaction is weak and the state is very shallow. For a dilute gas this contribution introduces a deviation from the ideal gas law that is larger than the correction due to the Fermi or Bose statistics of the particles. However, it is clear that 2D gases with a vanishing interparticle interaction strength should be well described by free Fermi or Bose gas models. This contradiction will be resolved in the present paper. A related question is what happens to the partition function when additional bound states appear with increasing strength of interaction between the particles. In the 3D case the answer to this question is based on a careful consideration of states in the continuum, which are modified by the interaction, i.e., taking scattering into account in the partition function calculation. ${ }^{4,5}$ The same approach should be applied in two dimensions; however, scattering theory in two dimensions is relatively undeveloped compared to the 3D case. For example, the relation between low-energy scattering and bound states, which has important consequences in the statistical mechanics, has been considered only very recently ${ }^{6,7}$ in two dimensions. In the present paper the connection between scattering and the statistical mechanics of a $2 \mathrm{D}$ plasma is studied.

In the next section we introduce the relation of the twodimensional scattering phase shift to the partition function and show that a proper consideration of the scattering states removes discontinuities in the partition function in an analogous fashion to the 3D case. In Secs. III and IV we explore this in more detail using an analytical model with an attractive square well potential before turning to a more realistic model of the statically screened electron-hole plasma, which is the main focus of this paper. We also compare in both cases the influence on the second virial coefficient of the exchange interaction and the screened direct interaction. Such considerations are crucial in understanding the nature of the strongly correlated electron-hole plasma in semiconductor quantum wells.

\section{PARTITION FUNCTIONS AND LEVINSON'S THEOREM}

The two-body interaction part of the partition function of 2D interacting Boltzmann particles is given by

$$
\begin{aligned}
Z_{\text {int }}= & \sum_{m, \nu} \exp \left(-E_{m, \nu} / k_{B} T\right) \\
& +\frac{1}{\pi} \int_{0}^{\infty} \sum_{m=-\infty}^{\infty} \frac{d \delta_{m}(q)}{d q} \exp \left(-q^{2} / q_{T}^{2}\right) d q,
\end{aligned}
$$

where $q_{T}^{2}=2 \mu k_{B} T / \hbar^{2}, \mu$ is the reduced mass, $m$ is the projection of the angular momentum onto the axis normal to the plane of $2 \mathrm{D}$ motion $(m=0, \pm 1, \pm 2, \ldots), \delta_{m}(q)$ is the $2 \mathrm{D}$ scattering phase shift ${ }^{8}$ dependent on the relative-motion momentum $q, E_{m, \nu}$ are the bound-state energies (index $\nu$ enumerates bound states with given $m$ ), and the double sum in the first term ranges only over bound states. Equation (1) is 
the 2D analogue of the Beth-Uhlenbeck formula and can be derived in the same fashion as in the $3 \mathrm{D}$ case. ${ }^{9}$

Often only the first term in Eq. (1) is considered when calculating an internal partition function, neglecting the phase shift term. For an attractive potential $g U(\rho)$, as $g$ decreases bound state energies increase towards the continuum. As such a state reaches zero energy, a partition function that contains only the bound state sum will be discontinuous. These unphysical singularities would extend to all the thermal properties, such as pressure and specific heat. Integrating by parts we can rewrite Eq. (1) as

$$
\begin{aligned}
Z_{\text {int }}= & \left\{\sum_{m, \nu} \exp \left(-E_{m, \nu} / k_{B} T\right)-\sum_{m=-\infty}^{\infty} \delta_{m}(0) / \pi\right\} \\
& +\frac{2}{\pi q_{T}^{2}} \int_{0}^{\infty}\left(\sum_{m=-\infty}^{\infty} \delta_{m}(q)\right) \exp \left(-q^{2} / q_{T}^{2}\right) q d q .
\end{aligned}
$$

For nonzero temperature the integral term in the right-hand side of Eq. (2) is a smooth function of the interaction strength $g$. If the phase shifts satisfy the condition

$$
\lim _{q \rightarrow 0} \delta_{m}(q)=\nu_{m} \pi,
$$

where $\nu_{m}$ is the number of bound states with given $m$, the zero-energy part of the phase shift integral in Eq. (1) exactly cancels the zero-energy part of the bound-state sum, removing the discontinuity in $Z_{\text {int }}$ as a function of the interaction strength. This cancellation is similar to the well-known behavior in three dimensions where the partition function discontinuities are removed ${ }^{5}$ with the help of Levinson's theorem. ${ }^{10}$ Equation (3) constitutes the 2D statement of Levinson's theorem.

As a central theorem of scattering theory, ${ }^{11}$ Levinson's theorem has been discussed for Dirac particles, multichannel scattering, multiparticle single-channel scattering, onedimensional scattering systems, impurities in AharonovBohm rings, systems with non-uniform effective mass, and even for time-periodic potentials. ${ }^{12}$ However, its applicability to the $2 \mathrm{D}$ scattering problem has been considered only recently. In Ref. 6 the 2D statement of Levinson's theorem, Eq. (3), was proposed and verified empirically, while in Ref. 7 this theorem was more rigorously established for cutoff potentials using the Green-function method. The above arguments, based on the partition function continuity requirement, provide an additional statistical mechanical justification of Levinson's theorem in 2D.

The two-body interaction part of the partition function can be used to calculate the second virial coefficient $B(T)$ that characterizes the first correction to the ideal gas law in the low-density expansion of pressure,

$$
P=n k_{B} T(1+B n+\cdots) .
$$

$B$ is positive for repulsive potentials, causing an increase in the pressure over its ideal-gas value, and negative for attractive potentials, causing a decrease in the pressure. A calculation of the second virial coefficient is meaningful in the dilute gas regime, where the mean inter-particle spacing $n^{-1 / 2}$ is larger than the thermal wavelength $\lambda$ $=\left(2 \pi \hbar^{2} / M k_{B} T\right)^{1 / 2}$ and the higher-order terms in Eq. (4) are negligible. For a free, Bose, or Fermi gas in two dimensions, $B(T)=\mp \lambda^{2} / 4,{ }^{13}$ the plus sign applying to the Fermi case, as the Pauli principle introduces an effective repulsion between fermions, thereby increasing the pressure. There is extensive ongoing research in the statistical mechanics of anyons, 2D particles obeying fractional statistics, ${ }^{14}$ and the second virial coefficient of a free anyon gas lies in between the bosonic and fermionic value. ${ }^{15}$ In this paper we focus on the relative importance of interaction-induced bound and scattering states on the second virial coefficient.

The $3 \mathrm{D}$ analysis of the second virial coefficient ${ }^{16}$ is easily reformulated for a $2 \mathrm{D}$ interacting gas. ${ }^{13}$ For a system of identical particles with spin $s$ the second virial coefficient is

$$
B^{(s)}(T)=\frac{\lambda^{2}}{2 s+1}\left(\mp \frac{1}{4}-\frac{2 Z_{\text {int }}^{(s)}}{2 s+1}\right),
$$

where the upper sign is for bosons and the lower sign for fermions. The exclusion principle modifies the sum over $m$ in Eq. (2) depending on the angular momentum parity, and the partition function [using Eq. (3)] is

$$
\begin{aligned}
Z_{i n t}^{(s)}= & (2 s+1) \sum_{m, \nu}\left(s+\frac{1 \pm(-1)^{m}}{2}\right) \\
& \times\left[\exp \left(-E_{m, \nu} / k_{B} T\right)-1\right]+(2 s+1) \frac{2}{\pi q_{T}^{2}} \\
& \times \int_{0}^{\infty} q d q\left[\sum_{m=-\infty}^{\infty}\left(s+\frac{1 \pm(-1)^{m}}{2}\right) \delta_{m}(q)\right] \\
& \times \exp \left(-q^{2} / q_{T}^{2}\right) .
\end{aligned}
$$

The electron-hole plasma constitutes a mixture of two components and for a binary mixture of components $C$ and $D$ having second virial coefficients $B_{C}^{(s)}$ and $B_{D}^{\left(s^{\prime}\right)}$ and densities $n_{C}$ and $n_{D}$, respectively, the second virial coefficient is $^{13}$

$$
B(T)=\left(\frac{n_{C}}{n}\right)^{2} B_{C}^{(s)}+2 \frac{n_{C} n_{D}}{n^{2}} B_{C D}+\left(\frac{n_{D}}{n}\right)^{2} B_{D}^{\left(s^{\prime}\right)} .
$$

In Eq. (7) $n=n_{C}+n_{D}$ and

$$
B_{C D}=-\lambda_{\mu}^{2} Z_{i n t},
$$

where

$$
\lambda_{\mu}^{2}=\frac{2 \pi \hbar^{2}}{2 \mu k_{B} T}, \quad \mu=\frac{M_{C} M_{D}}{M_{C}+M_{D}},
$$

and $Z_{\text {int }}$ is given by Eq. (1) or Eqs. (2) and (3) with the properly chosen reduced mass $\mu$.

\section{BOLTZMANN GAS WITH SHORT-RANGE ATTRACTION}

Our first example, the Boltzmann gas with weak shortrange attraction is chosen to elucidate how the second virial coefficient at given temperature vanishes when the binding energy $E_{b}$ decreases, even though the bound-state part of the internal partition function $Z_{\text {bound }}=\exp \left(E_{b} / k_{B} T\right)$ approaches unity rather than vanishes. To trace the precise nature of the 
cancellation of $Z_{\text {bound }}$ it is convenient to use Eq. (1) for $Z_{\text {int }}$ without the application of Levinson's theorem.

Let us assume that the 2D particles interact via an attractive square-well potential of radius $a$ and depth $V_{0}$. This simple model allows analytical treatment that provides insight into the generic behavior of a gas of attracting particles. To evaluate the partition function we need to analyze both the bound and scattering states in this potential. The binding energies for any value of angular momentum $m$ can be easily found by matching the logarithmic derivative of the radial wave function at $\rho=a$ :

$$
\frac{\sqrt{\kappa_{0}^{2}-\kappa^{2}} J_{|m|+1}\left(a \sqrt{\kappa_{0}^{2}-\kappa^{2}}\right)}{J_{|m|}\left(a \sqrt{\kappa_{0}^{2}-\kappa^{2}}\right)}=\frac{\kappa K_{|m|+1}(\kappa a)}{K_{|m|}(\kappa a)},
$$

where $\kappa_{0}^{2}=2 \mu V_{0} / \hbar^{2}, \kappa^{2}=2 \mu E_{b} / \hbar^{2}, J_{m}(x)$ is the Bessel function of the first kind and $K_{m}(x)$ is the modified Bessel function of the second kind. Note that for $\kappa_{0} a<2.4$ [i.e., smaller than the first root of $\left.J_{0}(x)\right]$ there is only one bound state (having $m=0$ ) and for $\kappa_{0} a<1$ this state is very shallow, e.g., for $\kappa_{0} a=1, E_{b} / V_{0} \approx 0.04$, for $\kappa_{0} a=0.5, E_{b} / V_{0}$ $\approx 2 \times 10^{-7}$. For a shallow $m=0$ state the transcendental equation for binding energies Eq. (10) reduces to

$$
\frac{\kappa_{0} a J_{1}\left(\kappa_{0} a\right)}{J_{0}\left(\kappa_{0} a\right)}=\frac{\kappa a K_{1}(\kappa a)}{K_{0}(\kappa a)}=-\frac{1}{\ln (c \kappa a)},
$$

where $c=\exp (\gamma) / 2(\gamma \approx 0.5772157 \ldots$ is Euler's constant).

For the unbound states with positive energy of the relative motion, $E=\hbar^{2} q^{2} / 2 \mu$, scattering phase shifts can be found in a similar fashion. For small values of the momentum, $q a$ $\ll 1$, all phase shifts for $m \neq 0$ are small compared to $\delta_{0}$ ( $s$-wave scattering) ${ }^{17}$ The tangent of the $s$-wave scattering phase shift for $q \ll \kappa_{0}$ (i.e., $E \ll V_{0}$ ) is given by: ${ }^{18}$

$$
\tan \delta_{0}=\frac{\pi / 2}{\ln (c q a)+\frac{J_{0}\left(\kappa_{0} a\right)}{\kappa_{0} a J_{1}\left(\kappa_{0} a\right)}} .
$$

Substituting $\kappa_{0} a J_{1}\left(\kappa_{0} a\right) / J_{0}\left(\kappa_{0} a\right)$ from Eq. (11) into Eq. (12) we get

$$
\tan \delta_{0}=\frac{\pi}{\ln \left(E / E_{b}\right)} .
$$

Note that this expression does not contain parameters of the potential $V_{0}$ and $a$ explicitly, and it is valid for an arbitrary potential well with a shallow $m=0$ level. ${ }^{19}$

Since the integrand in the partition function Eq. (1) contains an exponential factor $\exp \left(-q^{2} / q_{T}^{2}\right)$, the wave vectors $q$, which are larger than the thermal wave vector $q_{T}$, give negligible contribution to the value of the integral. Therefore for the short-range interaction or for low temperature, satisfying condition $k_{B} T \ll \hbar^{2} / 2 \mu a^{2}$, the scattering phase shifts need only be considered for $q \ll 1 / a$. Then all the terms in the phase shift sum in Eq. (1), except for the term with $m=0$, can be neglected. Finding the derivative of $\delta_{0}$ from Eq. (13) we obtain for the two-body interaction part of the partition function

$$
Z_{\text {int }}=\exp \left(E_{b} / k_{B} T\right)-\int_{0}^{\infty} \frac{\exp \left(-E / k_{B} T\right)}{\pi^{2}+\ln ^{2}\left(E / E_{b}\right)} \frac{d E}{E} .
$$

The integral in Eq. (14) is the Ramanujan integral, ${ }^{20}$ which can be rewritten as ${ }^{20,21}$

$$
\int_{0}^{\infty} \frac{e^{-x t}}{\pi^{2}+(\ln t)^{2}} \frac{d t}{t}=e^{x}-\nu(x),
$$

where

$$
\nu(x)=\int_{0}^{\infty} \frac{x^{t}}{\Gamma(t+1)} d t,
$$

with $x=E_{b} / k_{B} T$. Thus, the partition function acquires a very simple form

$$
Z_{\text {int }}=\nu\left(E_{b} / k_{B} T\right) .
$$

A similar result has been obtained recently for contactinteracting particles. ${ }^{14}$

To consider the small $x$ asymptotic of the function $\nu(x)$ it is convenient to expand the integral Eq. (16) in descending powers of $\ln (1 / x)$ :

$$
\nu(x)=\frac{1}{\ln (1 / x)}+\frac{\gamma}{\ln ^{2}(1 / x)}+O\left([\ln (1 / x)]^{-3}\right) .
$$

From Eq. (18) one can see that $Z_{\text {int }}$ and hence the second virial coefficient $B=-\lambda^{2} Z_{\text {int }}$ both vanish when $E_{b} / k_{B} T$ $\rightarrow 0$, although one bound state always exists. So the lowestorder density correction to the 2D ideal gas law vanishes only slowly as $1 / \ln \left(k_{B} T / E_{b}\right)$ as the binding energy is reduced. Note that, when the potential supports several bound states, the contribution of any shallow bound state with $m=0$ is cancelled by the scattering phase shift integral in the "logarithmic" manner described above. For $m \neq 0$ the cancellation has a power-law dependence in $E_{b} / k_{B} T{ }^{22}$ This implies that higher-order Levinson's theorems responsible for continuity of the partition function derivatives ${ }^{23}$ are different for $m$ $=0$ and $m \neq 0$, whereas the zeroth-order Levinson's theorem in two dimensions has the same form, Eq. (3), for all $m$.

For extremely weak interaction potential, such that $\left(\kappa_{0} a\right)^{2} \ll 1 / \ln \left(\hbar^{2} / 2 \mu a^{2} k_{B} T\right)$, from Eqs. (11) and (18) it follows that $Z_{\text {int }} \approx V_{0} \mu a^{2} / 2 \hbar^{2}$, which coincides with the perturbation theory result. In the other limit for large $x$ values $\left(E_{b} / k_{B} T \gg 1\right), \nu(x) \rightarrow e^{x},{ }^{24}$ therefore the exponential dependence of the partition function on the binding energy is recovered.

In Fig. 1 we plot the ratio of the total partition function $Z_{\text {int }}$ to its bound-state part $Z_{\text {bound }}=\exp \left(E_{b} / k_{B} T\right)$ as a function of $E_{b} / k_{B} T$. We do this for both the full expression, Eq. (16), and the first two terms in the asymptotic expansion, Eq. (18). One can see that the asymptotic expression (dashed line in Fig. 1) is accurate only for very small values of $E_{b} / k_{B} T$. Over a wide range of $E_{b} / k_{B} T$ both scattering and boundstate terms are important, e.g., when $E_{b}=k_{B} T$ the scattering term produces a $20 \%$ correction to $Z_{\text {int }}$. When $E_{b} / k_{B} T>3$ the bound-state contribution dominates completely.

It is instructive to compare the contributions to the second virial coefficient of the direct and the exchange interactions. For example, for a gas of spinless bosons the second virial 


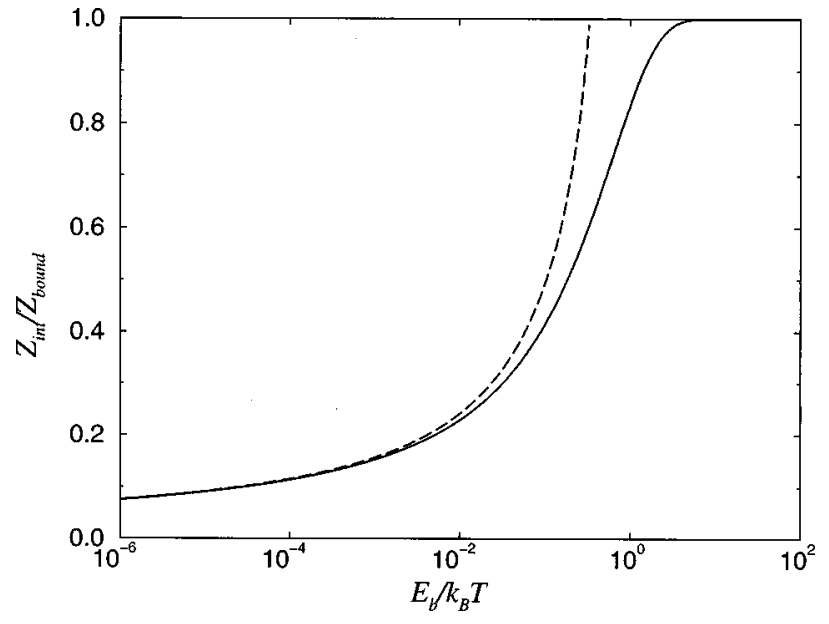

FIG. 1. Two-dimensional Boltzmann gas with a short-range weak attraction: the ratio of the two-body partition function to its bound-state part, $Z_{\text {int }} / Z_{\text {bound }}=\nu\left(E_{b} / k_{B} T\right) / \exp \left(E_{b} / k_{B} T\right)$, is plotted vs $E_{b} / k_{B} T$. Dashed line: the function $\nu(x)$ is approximated by $1 / \ln (1 / x)+\gamma / \ln ^{2}(1 / x)$.

coefficient is $B=-\lambda^{2}\left(1 / 4+2 Z_{\text {int }}\right)$. For $Z_{\text {int }}$ given by Eq. (17), the direct interaction term $\left(2 Z_{\text {int }}\right)$ is smaller than the exchange term (1/4) for small binding energies. Thus, as required for vanishing binding energy, the second virial coefficient is governed by the statistics of particles, despite the existence of a single bound state.

\section{ELECTRON-HOLE PLASMA}

In this section we study a more realistic model of an interacting gas than the one considered in Sec. III. We consider a mixture of the two types of Fermi particles, positively charged holes $(h)$ and negatively charged electrons $(e)$. This model is important for understanding the thermodynamic and optical properties of the electron-hole plasma in semiconductor quantum wells. In the low-temperature, low-density limit most electron-hole pairs are bound into excitons. This limit has been studied extensively because of the recent proposals of exciton condensates in this system..$^{25,26}$ The properties of the degenerate 2D electron-hole plasma are also well known. ${ }^{27}$ We consider the case when the temperature is comparable to the exciton binding energy so the occupation of continuum states (and therefore screening) is significant, although the carrier density is low enough to neglect the terms higher than $B n$ in the virial expansion. The temperature and density conditions under investigation are close to those in the regime where excitonic gain in wide-gap semiconductors is anticipated. ${ }^{28}$

We assume for simplicity equal masses $M_{e}=M_{h}=M$ and spins $s=s^{\prime}=1 / 2$ for both species. Then $Z_{h h}=Z_{e e}$ and the second virial coefficient for the mixture Eq. (7) acquires the form

$$
B=\frac{\lambda^{2}}{4}\left(\frac{1}{4}-2 Z_{e h}-Z_{e e}\right) .
$$

Here $\lambda=\lambda_{\mu}=\left(2 \pi \hbar^{2} / M k_{B} T\right)^{1 / 2}$ and the charge neutrality condition $n_{e}=n_{h}$ is taken into account.
The screened Coulomb attraction between electrons and holes is modeled using the Fourier representation of the interaction potential:

$$
V_{q}=-2 \frac{2 \pi}{q+q_{s}},
$$

where $q_{s}$ is the screening wave vector. Hereafter we employ 3D excitonic Rydberg units where length and energy are scaled, respectively, by the effective Bohr radius $a^{*}$ and Rydberg Ry*. For electron-electron and hole-hole repulsion the same potential with the opposite sign is used. Equation (20) is the well-known Thomas-Fermi expression for the Coulomb potential statically screened by a $2 \mathrm{D}$ electron gas. Being the long-wavelength static limit of the random-phase approximation, Eq. (20) is a simple model for the screened Coulomb potential in two dimensions. Nevertheless, this expression reflects the fact that the statically screened potential in two dimensions decreases at large distances slower than in the $3 \mathrm{D}$ case (as a power law rather than exponentially). Despite numerous more realistic corrections ${ }^{2,29}$ Eq. (20) remains the most widely used approximation for the 2D screening. ${ }^{30,31}$ This potential has been known for more than three decades ${ }^{8}$ but some of its unusual properties were only discovered recently, e.g., the existence of a remarkably simple relation between $q_{s}$ and the number of bound states. Namely, with decreasing screening, bound states appear at the critical integer values of the screening length given by the simple formula ${ }^{6}$

$$
\left(\frac{1}{q_{s}}\right)_{c}=\frac{(2|m|+\nu-1)(2|m|+\nu)}{2}, \quad \nu=1,2, \ldots,
$$

where $m$ is the angular momentum and $(\nu-1)$ indicates the number of nonzero nodes of the radial wave function. Equation (21) shows that several bound states corresponding to the given value of $(2|m|+\nu)$ appear simultaneously. This degeneracy is different from the degeneracy for the bound states of the unscreened 2D exciton (or hydrogen atom), for which the states with the same value of $(|m|+\nu)$ are degenerate..$^{30,32}$ The hidden symmetry that underlines this degeneracy has not been fully understood yet, and Eq. (21) still lacks a rigorous analytical derivation.

We also consider the low-density (nondegenerate) limit, when there is no Pauli blocking and the self-energy correction $^{33}$ to the Beth-Uhlenbeck formula can be neglected and Eqs. (2) and (3) and Eq. (6) can be used for $Z_{e h}$ and $Z_{e e}$, respectively. The shortcomings of this model for the quantitative description of a real system of photoexcited electrons and holes in semiconductor quantum wells are self-evident; however, it does provide a tractable model containing all the salient features of the system.

To find the second virial coefficient given by Eq. (19) one must calculate the binding energies and scattering phase shifts entering the partition functions $Z_{e h}$ and $Z_{e e}$. We use for this purpose the $2 \mathrm{D}$ modification of the variable-phase method $^{34}$ known from scattering theory. In this method the scattering phase shift and the function defining bound-state energies can be obtained as a large distance limit of the phase function, which satisfies the first-order, nonlinear Riccati equation originating from the radial Schrödinger equation. The variable-phase method application to scattering and 


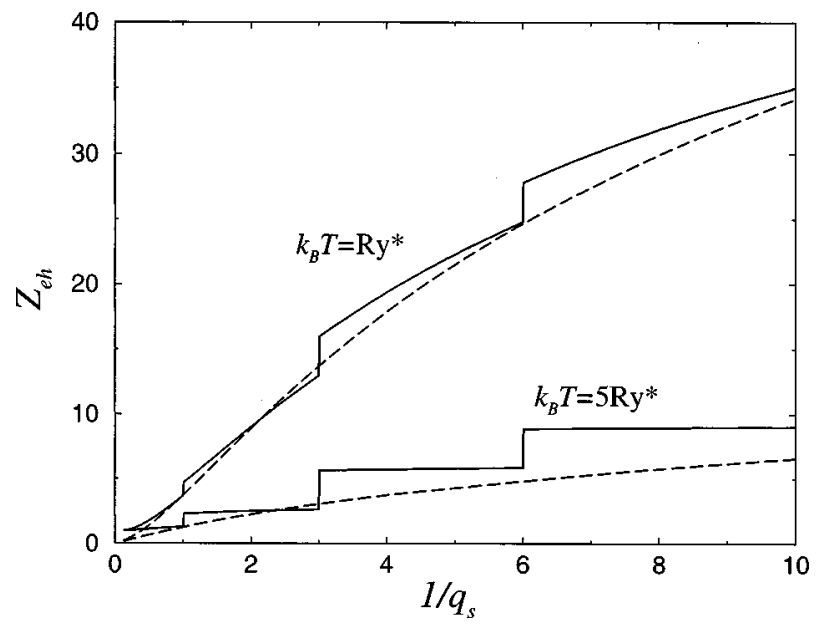

FIG. 2. The electron-hole part of the partition function $Z_{e h}$ vs the screening length $1 / q_{s}$ for two values of $k_{B} T /$ Ry. Solid lines show the bound-state contributions $Z_{\text {bound }}$ only. Dashed lines: $Z_{\text {bound }}+Z_{\text {scatt }}$.

bound states in the screened Coulomb potential (20) is described in detail in Ref. 6. The method is especially effective for calculation of shallow-state binding energies and lowenergy scattering phase shifts.

Figure 2 shows the results from the calculation of the electron-hole part of the partition function $Z_{e h}$, which contains both the bound state sum and the scattering phase shift integral. In this figure $Z_{e h}$ is plotted as a function of the inverse screening wave number $1 / q_{s}$. For the electron-hole plasma $q_{s}$ is a function of carrier density, ${ }^{30}$ and in the purely 2D case $q_{s} \propto\left(n_{e}+n_{h}\right)$ for low densities and is independent of density, $q_{s} \rightarrow 8 / a^{*}$ for $M_{e}=M_{h}$, in the degenerate limit. Here we treat $q_{s}$ as a parameter characterizing the strength of the screened interaction potential Eq. (21). To emphasize the role of scattering we show on the same plot the bound-state sum, $Z_{\text {bound }}=\Sigma_{m, \nu} \exp \left(-E_{m, \nu} / k_{B} T\right)$, which exhibits jumps whenever new bound states appear [i.e., when $q_{s}$ satisfies Eq. (21)]. These jumps become higher with increasing screening length $1 / q_{s}$ since several bound states appear simultaneously. As can be seen, the additional scattering phase shift contributions completely remove these jumps. The partition function is plotted for two values of the ratio of $k_{B} T$ to the excitonic Rydberg, $k_{B} T=1 \mathrm{Ry}^{*}$ and $k_{B} T=5 \mathrm{Ry}^{*}$, which roughly correspond to $\mathrm{ZnSe}$ (or $\mathrm{GaN}$ ) and $\mathrm{GaAs}$ at room temperature. One can see that for high temperature (or low binding energy) the bound-state contributions to $Z_{e h}$ are suppressed by the scattering phase shift integral more strongly than in the $k_{B} T=1 \mathrm{Ry}^{*}$ case.

In Fig. 3 the second virial coefficient $B$ (scaled by $\lambda^{2}$ ) is plotted versus the screening wave number $q_{s}$ for two different values of $k_{B} T / \mathrm{Ry}^{*}$. Equation (19) is used for the calculation of $B$, and the repulsion $\left(Z_{e e}\right)$ term partially compensates the $Z_{e h}$ term. This compensation is especially significant in the high-temperature case $k_{B} T=5 \mathrm{Ry}^{*}$, in which the $2 \mathrm{D}$ electron-hole plasma behaves much like an ideal gas over a wide range of screening wave vectors. For $k_{B} T=1 \mathrm{Ry}^{*}$ the electron-hole attraction term dominates and the plasma is strongly correlated for all values of $q_{s}$. In this case a small statistical repulsion $\left(B / \lambda^{2}=1 / 16\right.$, horizontal line in Fig. 3), which is due to the fermionic nature of elec-

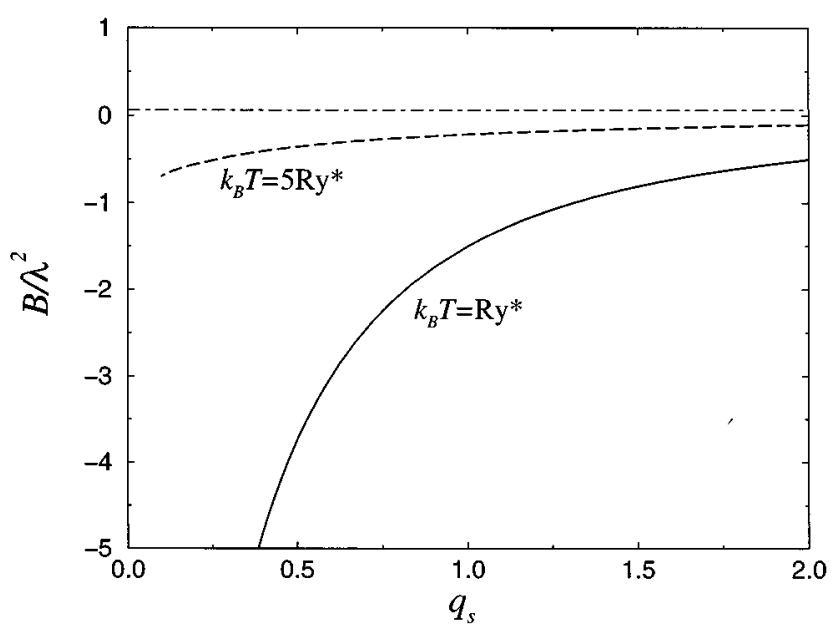

FIG. 3. The second virial coefficient of the dilute electron-hole plasma $B / \lambda^{2}$ as a function of the screening wave number $q_{s}$. Solid line, $k_{B} T=1$ Ry*; dashed line, $k_{B} T=5 \mathrm{Ry}^{*}$; dot-dashed line, noninteracting dilute electron-hole plasma, $B / \lambda^{2}=1 / 16$.

trons and holes, can be completely neglected. Thus, at room temperature the electron-hole plasma in GaAs-based quantum wells can be treated as an ideal gas, whereas in wide-gap semiconductors (e.g., $\mathrm{ZnSe}$ or $\mathrm{GaN}$ ) due to the high value of Ry* the 2D electron-hole plasma is strongly correlated and excitonic effects are important for its thermodynamic properties.

\section{CONCLUSION}

In this paper we show that a proper account of scattering eliminates discontinuities in thermodynamic properties of the nonideal 2D gas whenever extra bound states appear with small increase of the strength of an attractive potential. This treatment provides a heuristic proof of Levinson's theorem in two dimensions.

We trace the way in which the bound-state contribution to the partition function of the 2D gas, with a weak short-range attraction between its particles, vanishes when the binding energy decreases. A weak $1 / \ln \left(k_{B} T / E_{b}\right)$ binding energy dependence of the second virial coefficient of such a gas is found for $E_{b} / k_{B} T \rightarrow 0$

A consistent treatment of bound and scattering states in a screened Coulomb potential allows us to calculate the quantum-mechanical second virial coefficient of the dilute 2D electron-hole plasma and to establish the difference between the nearly ideal electron-hole gas in GaAs and the strongly correlated exciton/free-carrier plasma in wide-gap semiconductors.

The 2D electron-hole plasma was considered in the lowdensity nondegenerate limit only. Transition to the strongly degenerate Fermi limit and related questions of Pauli blocking and self-energy corrections to the Beth-Uhlenbeck formula in 2D remain the subject of further research.

\section{ACKNOWLEDGMENTS}

This work was supported by the U.K. EPSRC and the Royal Society. 
*Also at A. F. Ioffe Physico-Technical Institute, St. Petersburg, Russia.

${ }^{1}$ The Quantum Hall Effect, edited by R. E. Prange and S. M. Girvin (Springer, New York, 1990).

${ }^{2}$ T. Ando, A. B. Fowler, and F. Stern, Rev. Mod. Phys. 54, 437 (1982).

${ }^{3}$ W. W. Chow, S. W. Koch, and M. Sargent III, SemiconductorLaser Physics (Springer, Berlin, 1994).

${ }^{4}$ A. I. Larkin, Zh. Éksp. Teor. Fiz. 38, 1896 (1960) [Sov. Phys. JETP 11, 1363 (1960)].

${ }^{5}$ F. J. Rogers, H. C. Graboske, Jr., and H. E. DeWitt, Phys. Lett. 34A, 127 (1971); F. J. Rogers, Phys. Rev. A 4, 1145 (1971).

${ }^{6}$ M. E. Portnoi and I. Galbraith, Solid State Commun. 103, 325 (1997).

${ }^{7}$ Q.-G. Lin, Phys. Rev. A 56, 1938 (1997).

${ }^{8}$ F. Stern and W. E. Howard, Phys. Rev. 163, 816 (1967).

${ }^{9}$ E. Beth and G. E. Uhlenbeck, Physica (Amsterdam) 4, 915 (1937); see also, e.g., L. D. Landau and E. M. Lifshitz, Statistical Physics (Pergamon, New York, 1980), Pt. 1, p. 236.

${ }^{10}$ N. Levinson, K. Dan. Vidensk. Selsk. Mat. Fys. Medd. 25, 3 (1949).

${ }^{11}$ R. G. Newton, Scattering Theory of Waves and Particles (McGraw-Hill, New York, 1966).

${ }^{12}$ Zh.-Q. Ma, Phys. Rev. Lett. 76, 3654 (1996); N. Poliatzky, ibid. 76, 3655 (1996); F. Vidal and J. Letourneaux, Phys. Rev. C 45, 418 (1992); L. Rosenberg and L. Spruch, Phys. Rev. A 54, 4978 (1996); 54, 4985 (1996); K. A. Kiers and W. van Dijk, J. Math. Phys. 37, 6033 (1996); M. S. Debianchi, ibid. 35, 2719 (1994); V. Milanović et al., Phys. Lett. A 170, 127 (1992); V. Milanović et al., J. Phys. A 25, L1305 (1992); M. DiVentra et al., Phys. Rev. B 55, 1353 (1997); P. A. Martin and M. S. Debianchi, Europhys. Lett. 34, 639 (1996), and references therein.

${ }^{13}$ R. L. Siddon and M. Schick, Phys. Rev. A 9, 907 (1973); J. G. Dash, Films on Solid Surfaces (Academic, New York, 1975).

${ }^{14}$ P. Giacconi, F. Matoni, and R. Soldati, Phys. Rev. B 53, 10065 (1996), and references therein.

${ }^{15}$ P. Arovas, R. Schrieffer, F. Wilczek, and A. Zee, Nucl. Phys. B 251, 117 (1985). For a review on anyons see, e.g., Fractional Statistics and Anyon Superconductivity, edited by F. Wilczek (World Scientific, Singapore, 1990).

${ }^{16}$ J. E. Kilpatrick, J. Chem. Phys. 21, 274 (1953); J. E. Kilpatrick, W. E. Keller, E. F. Hammel, and N. Metropolis, Phys. Rev. 94, 1103 (1954).
${ }^{17}$ L. D. Landau and E. M. Lifshitz, Quantum Mechanics (Nonrelativistic Theory) (Butterworth-Heinemann, Oxford, 1997).

${ }^{18}$ M. E. Portnoi, Pis'ma Zh. Tekh. Fiz. 14, 1252 (1988) [Sov. Tech. Phys. Lett. 14, 547 (1988)].

${ }^{19}$ It is interesting to note that the expression for the scattering cross section for resonant $s$-wave scattering in two dimensions, $\sigma$ $=(4 / q) \sin ^{2} \delta_{0}=\left(4 \pi^{2} / q\right) /\left[\pi^{2}+\ln ^{2}\left(E / E_{b}\right)\right]$, despite being quite different from its $3 \mathrm{D}$ equivalent (Ref. 17), nevertheless also has a pole at $E=-E_{b}$.

${ }^{20}$ G. H. Hardy, Ramanujan (Cambridge University Press, Cambridge, England, 1940), p. 196.

${ }^{21}$ I. S. Gradshtein and I. M. Ryzhik, Tables of Integrals, Series, and Products (Academic Press, New York, 1994).

${ }^{22}$ M. E. Portnoi and I. Galbraith (unpublished).

${ }^{23}$ D. Bollé, Ann. Phys. (N.Y.) 121, 131 (1979).

${ }^{24}$ Higher Transcendental Functions, edited by A. Erdélyi, (McGraw-Hill, New York, 1955), Vol. 1, p. 221.

${ }^{25}$ X. Zhu, P. B. Littlewood, M. S. Hybertsen, and T. M. Rice, Phys. Rev. Lett. 74, 1633 (1995).

${ }^{26}$ J. Fernández-Rossier and C. Tejedor, Phys. Rev. Lett. 78, 4809 (1997), and references therein.

${ }^{27}$ S. Schmitt-Rink, D. S. Chemla, and D. A. B. Miller, Adv. Phys. 38, 89 (1989).

${ }^{28}$ I. Galbraith, in Microscopic Theory of Semiconductors: Quantum Kinetics, Confinement and Lasers, edited by S. W. Koch (World Scientific, Singapore, 1996), p. 211, and references therein.

${ }^{29}$ C. Bulutay and M. Tomak, Phys. Rev. B 54, 14643 (1996); K. Schrüfer et al., Superlattices Microstruct. 21, 223 (1997); A. Gold and A. Ghazali, J. Phys.: Condens. Matter 9, 6885 (1997), and references therein.

${ }^{30} \mathrm{H}$. Haug and S. W. Koch, Quantum Theory of the Optical and Electronic Properties of Semiconductors (World Scientific, Singapore, 1994).

${ }^{31}$ J. A. Reyes and M. del Castillo-Mussot, Phys. Rev. B 56, 14893 (1997), and references therein.

${ }^{32}$ S. Flügge and H. Marschall, Rechenmethoden der Quantentheorie (Springer, Berlin, 1952), Problem 24.

${ }^{33}$ R. Zimmermann and H. Stolz, Phys. Status Solidi B 131, 151 (1985).

${ }^{34}$ F. Calogero, Variable Phase Approach to Potential Scattering (Academic, New York, 1967). 